\title{
Research on Virtual Laboratory Platform Architecture Design Based on Internet
}

\author{
Jing Zhang ${ }^{1, a}$ Xin $\mathrm{Pu}^{2, \mathrm{~b}}$ \\ ${ }^{12}$ College of Optical and Electronical Information, Changchun University of Science and \\ Technology, Changchun, Jilin, China, 130012 \\ aemail, ${ }^{b}$ email
}

Keywords: Virtual Laboratory Platform, Architecture Design, Internet

\begin{abstract}
With the deepening reform and expanding college enrollment in China's higher education system, the traditional experimental teaching has been unable to meet the needs of today's higher education development. Based on the original network engineering laboratory, based on the introduction of virtual simulation technology to build a virtual network based on hybrid technology prototype experimental system engineering and web technology, we designed an online self-learning, virtual laboratory system self-test and experimental operation and management. The system ensures that the learner at any time, any place can get real-time learning and guidance, in order to achieve the goal of learning networking skills.
\end{abstract}

\section{Introduction}

With the rapid development and application of Internet, the modern distance education led to a profound change in the mode of education and educational concept of network distance learning has become an overseas university, an important mode of teaching in secondary schools. Research and development of distance education and the need to establish develop rich distance learning resources, including teaching content information and courseware production and development, the establishment of virtual laboratories, etc. to accept remote virtual laboratory to provide an education for students regardless of time and place, laboratory equipment limits of experimental environment. Many of the world famous universities, such as Oxford and Carnegie Mellon University have developed a virtual laboratory. Some prototype virtual lab has Internet users open to international, such as the University of Oxford new chemical information industry center allowing students over the Internet in a virtual experiment room interactive chemistry experiments;. Carnegie Mellon University offers an online analysis on characteristics of the circuit and motor control experiments of virtual experiments in virtual terms, has not yet built can use virtual lab platform, virtual laboratory has been built mainly to provide appropriate experimental courses, to facilitate students' learning or understanding for scientific research, such as the evaluation of Qos routing algorithms, online expert systems, etc. in interactivity, scalability and reusability of software aspects of some shortcomings, such as the students cannot customize the test, you cannot own development laboratory equipment added to the lab extension lab, difficult to reuse existing modules to construct a new experiment. To solve these problems, the authors proposed architecture configured virtual laboratory platform, on this basis, to how components provide different courses of laboratory equipment to construct the corresponding laboratory.

\section{The Concept and Research Development of Virtual Laboratory}

The concept of virtual laboratory was first used by a professor William Wolff University of Virginia in 1989 to the United States proposed, which describes a computer network virtual lab environment. Network engineering virtual laboratory is a member of the family of the virtual laboratory and it is a virtual experiment system study, design, management and research for the network theory, network engineering and network security. Subject Network Virtual Laboratory under the experimental network engineering architecture, the use of virtual simulation technology, network technology and software technology, computer technology, engineering experiment to create a virtual environment, 
running a variety of real or virtual network device to set up experimental topology design and management, constitute new teaching experiments. Network engineering virtual laboratory is an expansion and extension of the traditional network engineering experiment teaching. It is good to meet learners' individual learning needs and self-learning to ensure that the learner at any time, any place can get real-time learning and guidance, in order to better achieve the goal of learning networking skills.

Virtual Laboratory also known as "co-lab " was first used to describe a computer in a network of virtual lab environment. It is committed to building a synthesis of different tools and techniques of electronic, network integration of scientific research environment. In this environment, the scientists can be very efficient use of geographically distributed resources (data, information, equipment and manpower) to engage in research activities.Wulf vividly called virtual Lab. research center without walls. In the meantime, researchers regardless of their geographical distribution, were able to jointly research - interaction with colleagues, instrumentation, sharing data and resources, access to information in a digital library, a study co-authored the report and all these activities do not have to be face to face, but in a distributed network environment support, by means of e-mail systems, multimedia conferencing systems to achieve. Therefore, the virtual laboratory is essentially a distributed computer system. In the system, the configuration having a remote control telemetry capable network equipment research and data collection platform, there are a variety of tools to support collaborative activities, built to support large-scale data sharing digital library. All technical support are designed to enhance the virtual laboratory scientists, instruments and equipment blending and interaction between data and other resources to improve research efficiency, reduce research costs, and provide a strong technical support for the development of high-tech science and technology, and ultimately promote the progress of human society.

Currently, there are many domestic and international organizations have already carried out research and construction of virtual experiment system, especially in some well-known foreign universities, there are more built and put into use virtual experiment system, covering computer network, mathematics , artificial intelligence, life sciences, chemistry, physics, biology, engineering, communications, 3DCAD, graphic images, agricultural science teaching and research in the field.

VSL Acronym Virtual Systems Laboratory was founded in 1989, established by the Education and Training Institute Gentra1 Florida University, which aims to raise artistic expression and improved computer graphics simulation of the human-computer interface design. Currently, the lab is carrying out a number of research and support of virtual experiments related technologies, real-time physics simulation technology, low-cost technologies such as virtual graphics simulation experiment environment networks and parallel computing, complex experimental environment, etc; a lot has been made research, such as the design and development of the first case of real-time dynamic virtual environment, the first case of VR3DCAD virtual design system.

\section{Architecture Design of Virtual Laboratory}

Network Virtual Laboratory is a heterogeneous solution to the problem, no matter where students can experiment. It requires participation experimenter shared experimental environment and experimental requirements. Web technology will undoubtedly provide a platform for long network engineering virtual laboratory infrastructure, which we need to first solve the structural problems of the system.

Based On C/S Two-Tier Structure. Client / Server C structure appeared earlier, a wide range of applications a two-tier structure, C / S structure of the rational allocation of tasks to the client and server side, front and calculate separation, thereby reducing the cost of communications systems can take advantage of both ends of the hardware environment. Because under C / S structure of the application must be installed to each client, a dynamic library file system database must also reside on the client, which gives the system updates, upgrades and maintenance to bring a lot of trouble. And application system c / s structure scalability, maintainability and safety are poor, which makes applications C / S structure is subject to many restrictions.

Based On B / S Three-Tier Structure. Browser / Server structure, the C / S structure, client 
program reduced to a common browser software, which the C / S structure of the server is decomposed into a database server and a Web server, thereby constituting a three-tier B / S architecture. B / S structure greatly simplifies the client's work and the server will take on more work. Clients use the browser, requesting access to the database to the Web server via HTTP protocol, Web server accepts a client request, this request into SQL syntax to the database server, the database server gets the request, verify its legitimacy and data processing, and then the results back to the Web server, Web server once all the results obtained will be transformed into the form of an HTML document forwarded to the client browser, a friendly Web page form is displayed.

Through the above C / S and B Comparative Analysis / S structure of the two systems, based on what we want to develop a virtual laboratory design requirements, we chose the B / S three-tier structure to develop network engineering virtual laboratory system. The system includes a client application Web server, run the server emulator three parts, as shown in FIG. Web application server handles client requests experimental network topology configuration, complete the communication transfer between the client and the server running the simulator. Run the server software using GNS3 simulator simulates the actual device topology generation parameters required by the application using the Web server, management and maintenance needs analog equipment.

\section{Implementation Technology of Client}

Virtual Lab client uses an object-oriented design methods and component technology, the Java language, in a manner embedded in the HTML file Applet program available to users having nothing to do with Java programs internal platform, secure, robust, etc. only need to support Java-enabled browser to run. the client virtual laboratory designed to meet a digital image processing, digital communication theory, digital signal processing requirements common platform for multiple test courses, each of the specific features of each course way algorithm laboratory equipment or a component of the package, to create a dynamic laboratory component objects, set object properties, call the object method, event response object, so that the components communicate with each other to provide a mechanism to allow users to use a combination of components corresponding experimental manner .

Reflection Technology of Java Language. With the increase of laboratory equipment, a sharp increase in the number of files, but some functions are some users do not need or use of a particular experiment. In order to avoid unnecessary file transfer, the successful laboratory equipment package independent of JavaBean components to reduce the size of each file. Using Java language reflection function, the object factory design model, JavaBean container construction, the introduction of dynamic load and create objects. Therefore, only when the user needs to download the device class file. In this mode, the system has good scalability, can introduce user-defined laboratory equipment and experimental treatment. Lab provides the capability to register a custom class file and class file by location registration (URL Notation) processing method and class, the experimenter will be able to properly use the laboratory equipment.

More Threading Technology. Multi-threading technology has the following advantages: a1 generated responsive UI during the experiment is running, when a large amount of laboratory equipment operation or transfer large data files on the Internet, take a long time to process, single-threaded. The program causes the system cannot be timely response to user actions and cannot understand the intermediate state or interrupt the running experiment, and multithreaded programs can effectively solve these problems .b1 parallel processing to speed up the processing speed. when using distributed processing, multi-processor system, or an experimental device appears IPO obstruction, the use of multi-threaded program that allows data laboratory equipment unrelated to run in parallel to speed up the operation speed dynamic visual effects .c1 analog data transmission, etc. as the network simulation experiments, more communication ports simultaneously transmit signals to each other; communication principle experiment, oscilloscope accepts multiple signal sources.

XML Technology. Use XML (Extend Mark Language) to save the system configuration parameters such as the system comes with laboratory equipment .XML good structural features and 
scalability, easy to build any complex hierarchical data models, the new Custom symbols of.

Plug-In Technology. The rapid development of Java to make JDK (Java Developer Kit, the Java Development Kit) version update faster, currently at version JDK1.3, JDK1.4 new beta version has also been published, the browser version of the update speed is relatively in order to slow the high version of the Java program can run on older versions of the browser, Sun has provided its solutions: the plug-in version of the Java virtual machine high into the browser, so that the high version of the JDK code the browser can be interpreted as a virtual laboratory developed using JDK1.3, can install the appropriate plug-in IE or NetScape6.0 following version, it can make the program run smoothly. Plug-in technology can also be configured to use a separate buffer for the Applet the buffer zone is different from the browser's buffer whose content will not be replaced with other documents such as html, etc., which can effectively avoid duplication download the Applet.

\section{Conclusion}

With the rapid development of computer technology and virtual simulation technology, building a virtual network engineering laboratory has become an inevitable trend in the development of relevant professional experiment teaching of Universities network technology. This not only provides good conditions for independent learning, can also have a profound impact on improving the experimental environment, optimize the teaching process.

\section{Acknowledgements}

Fund Project: Provincial Project: Virtual Simulation Center (Yoshitaka learn numbers [2015] No. 7)

\section{References}

[1] Jia Xinzhang, Li Jingyuan. Technology Review, Vol. 6 (2004) No 53, p.25-26

[2] Wang Qunyong. Computer Engineering and Science, Vol. 12 (2005) No 27, p.74-76

[3] Qian Xiyuan, Jing Jianfen. Computer Engineering, Vol. 30 (2004) No 19, p.144-145

[4] Wang Kuailiang. Microcomputer Applications, Vol. 29 (2008) No 27, p.21-23

[5] Zhang Gongxu, Sun Jing. New Quality Management, Vol. 8 (2003) No 27, p.57-60 